Junfang Xian

Zhengyu Zhang

Zhenchang Wang

Jing Li

Bentao Yang

Qinghua Chen

Qinglin Chang

Liyan He

\section{Evaluation of MR imaging findings differentiating cavernous haemangiomas from schwannomas in the orbit}

Received: 10 December 2009

Revised: 5 February 2010

Accepted: 10 February 2010

Published online: 15 April 2010

(C) The Author(s) 2010

This article is published with open access at Springerlink.com
J. Xian $(\bullet) \cdot$ Z. Zhang $\cdot$ Z. Wang $\cdot$ J. Li $\cdot$ B. Yang $\cdot$ Q. Chen $\cdot$ Q. Chang $\cdot$ L. He Department of Radiology, Capital Medical University, Beijing Tongren Hospital, Beijing 100730, China e-mail: cjr.xianjunfang@vip.163.com Tel.: +86-108-5111724

\begin{abstract}
Objective It is important to distinguish between orbital cavernous haemangioma and schwannoma because the treatments of choice for the two tumours are different. The aim was to evaluate MR imaging findings distinguishing the two tumours. Methods Magnetic resonance imaging including T1- and T2-weighted imaging and contrast-enhanced MR imaging was performed in 43 patients with cavernous haemangiomas and 16 patients with schwannomas confirmed by pathology. Location, configuration, margins, signal intensity, homogeneity and enhancement pattern of the tumour were retrospectively evaluated. Results There was a significant difference between cavernous haemangiomas and schwannomas regarding the location, configuration and margins of the mass, signal intensity and homogeneity on T1- and T2-weighted imaging, the spread pat-
\end{abstract}

tern of contrast enhancement, the enhancement pattern and the type of time-intensity curve $(P<0.05)$. Markedly homogeneous hyperintensity signal on $\mathrm{T} 2$-weighted imaging and the spread pattern of the contrast enhancement favoured cavernous haemangioma rather than schwannoma $(P<0.01)$. Conclusion Cavernous haemangiomas and schwannomas have different MR imaging features that could be helpful in the differentiation between the tumours. The spread pattern of the contrast enhancement on dynamic contrast-enhanced MR imaging is the most reliable finding distinguishing cavernous haemangiomas from schwannomas.

Keywords Orbit - Cavernous haemangioma $\cdot$ Schwannoma . Magnetic resonance imaging . Differential diagnosis

\section{Introduction}

Cavernous haemangioma is the most common primary orbital tumour in adults, accounting for $6 \%$ of all orbital lesions [1-3]. Orbital schwannomas are benign peripheral nerve sheath tumours and constitute $1 \%$ of all orbital tumours [1, 3]. Orbital cavernous haemangiomas and schwannomas present as slow progressive enlargement and the most common presenting sign and symptom is painless proptosis [4-8]. Although both orbital cavernous haemangiomas and schwannomas share many similar clinical and radiological characteristics [5-8], it is impor- tant to distinguish between the tumours because they demonstrate important different features and the treatments of choice for the two are different [3, 7-9].

Magnetic resonance imaging, especially dynamic MR imaging, plays a crucial role in the differentiation of orbital cavernous haemangiomas and schwannomas $[7,8$, 10-14]. The spread pattern of the contrast enhancement on dynamic contrast-enhanced MR imaging is considered the most important finding distinguishing the two tumours [10-14]. However, the literature on MR imaging findings in the differentiation of these two tumours is based on small patient populations [12]. Consequently, this study 
aims to assess MR imaging findings distinguishing orbital cavernous haemangiomas from schwannomas and to elaborate on specific differences between the two tumours.

\section{Materials and methods}

\section{Patient population}

Between June 2004 and February 2009, 43 patients with cavernous haemangiomas and 16 patients with schwannomas confirmed by pathological results were enrolled in the protocol. MR imaging including T1- and T2-weighted imaging and contrast-enhanced MR imaging was performed in all 59 patients. Most patients presented with painless insidious proptosis. Some patients demonstrated a limitation of extraocular motion, diplopia and strabismus.

Informed consent was obtained from all patients for anonymous performance of radiological studies and analysis of clinical data. Our institutional review board approved the study protocol.

\section{MR imaging}

Magnetic resonance imaging was performed on a General Electric (GE) Signa 1.5-T MR imaging system (GE Healthcare, Milwaukee, WI, USA) with an eight-channel head coil.

MR imaging was performed with T1-weighted (repetition time/echo time, $400 / 15 \mathrm{~ms})$ and T2-weighted $(3,000 /$ 120) fast spin-echo images. A $16-\mathrm{cm}$ field of view, a 3mm-thick section at 0 interval, and a $288 \times 256$ matrix with two signals acquired were used. Dynamic contrastenhanced (DCE) MR imaging was performed by using a fast spoiled gradient recalled (FSPGR) sequence with 8.4/ $4 \mathrm{~ms}$, a flip angle of $15^{\circ}$, one excitation, a matrix of $256 \times$ 160 , a field of view of $220 \times 220 \mathrm{~mm}$, and a section thickness of $3.2 \mathrm{~mm}$ at 0 interval. A power injector (Medrad, Indianola, PA, USA) with an injection flow rate of $2 \mathrm{~mL} / \mathrm{s}$ was used. The acquisition of the dynamic images began concurrently with the initiation of the injection of $0.1 \mathrm{mmol}$ of gadopentetate dimeglumine (Magnevist, Schering, Berlin, Germany) per kilogram. The contrast material injection was followed by a $20-\mathrm{mL}$ saline flush. Each MR sequence yielded 12 anatomical slices in $13 \mathrm{~s}$; this was repeated 12 times with an interval between each sequence of $12 \mathrm{~s}$. Total imaging time was $288 \mathrm{~s}$ (4 min $48 \mathrm{~s})$.

DCE MR imaging was evaluated by using a GE AW 4.2 workstation (GE Healthcare). A region of interest (ROI) was drawn manually on the dynamic images for assessment of the enhancement kinetics. We chose the area that demonstrated the greatest degree of early enhancement so that the time-intensity curve (TIC) could be generated. The area of the ROI typically was $8 \mathrm{~mm}^{2}$. The contrast index $(\mathrm{CI})$ was calculated from: $\mathrm{CI}=[$ (signal intensity post-contrast)-(signal intensity pre-contrast)]/ (signal intensity pre-contrast). Tpeak was the time to peak enhancement. The TICs were analysed qualitatively as wash-out, persistent or plateau-shaped curves.

\section{Image interpretation}

Magnetic resonance imaging features including DCE MR imaging were retrospectively evaluated by an experienced head and neck radiologist (15 years' experience). Location, configuration, margins, signal intensity, homogeneity and enhancement pattern of the tumour were assessed. The location was defined as anterior orbit (anterior orbit is defined as the orbit anterior to the posterior ethmoidal artery), posterior orbit (posterior orbit is defined as the orbit posterior to the posterior ethmoidal artery), or both anterior and posterior orbit; extraconal or intraconal space; superior or inferior orbit; and nasal or temporal orbit. The configuration was defined as round, ovoid or irregular. The margin was defined as smooth or lobulated. On contrast-enhanced MR imaging including DCE MR imaging, the spread pattern of the contrast enhancement, enhancement pattern, CI and type of TIC were evaluated. The spread pattern of the contrast enhancement was divided into two groups: one starting from a small point or portion and the other starting from a wide area. "Progressive" enhancement means that on early imaging after the injection, one small point or portion of enhancement was initially noted, then contrast media progressively filled up the tumour and eventually the mass showed total and homogeneous enhancement (Fig. 1) [13]. Other patterns of enhancement included gradually heterogeneous enhancement and gradually homogeneous enhancement.

\section{Statistical analysis}

Frequency distribution of individual MR imaging features in cavernous haemangiomas was compared with that in schwannomas with Chi-squared tests. Fisher's exact test was performed when the sample size in the subgroups was deemed too small. A $P$ value of less than 0.05 was considered to represent a significant difference.

\section{Results}

Tables 1 and 2 describe the frequency distribution of nonenhanced MR imaging features (Table 1) and contrastenhanced MR imaging features (Table 2). There was a significant difference between cavernous haemangiomas and schwannomas regarding the location (including anterior orbit, posterior orbit, or both anterior and posterior orbit; extraconal or intraconal space), configuration and margins of the mass, signal intensity and 

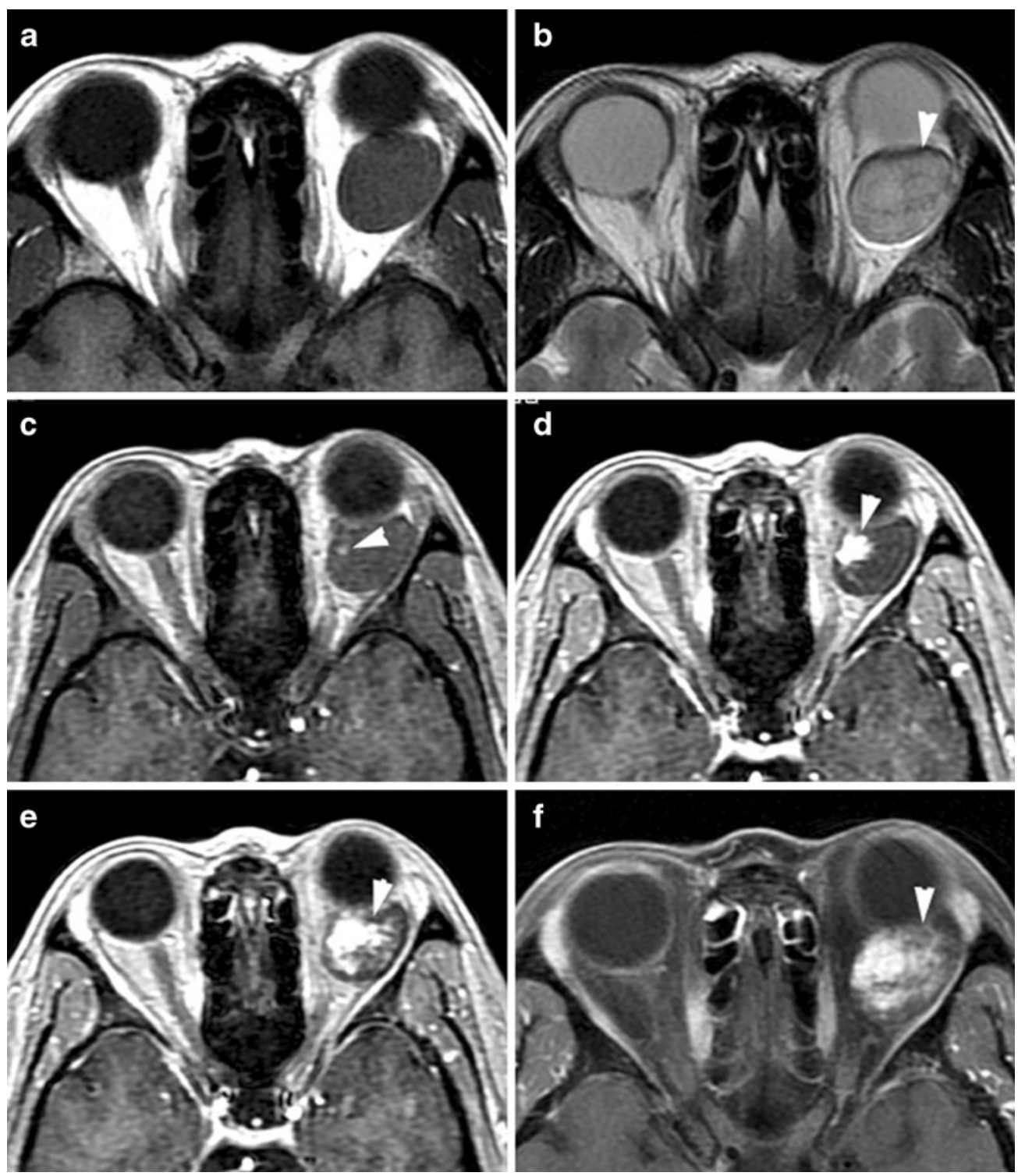

Fig. 1 A 54-year-old woman with a cavernous haemangioma. a Axial T1-weighted imaging demonstrated an oval intraconal isointense tumour relative to muscle. b The tumour appeared hyperintense (arrowhead) relative to muscle on axial T2-weighted imaging. c-e Dynamic MR images obtained at 25, 75 and 225 s,

homogeneity on T1- and T2-weighted imaging, the spread pattern of the contrast enhancement, enhancement pattern and type of time-intensity curve (TIC) $(P<0.05)$ (Figs. 1, $2,3)$.

Orbital cavernous haemangiomas were always a welldefined, ovoid, intraconal mass with markedly homogeneous hyperintensity signal on T2-weighted imaging. Schwannomas often demonstrated a heterogeneously isointense or mildly hyperintense mass in the intraconal or extraconal space.

After gadolinium administration, all 43 cavernous haemangiomas showed enhancement starting from a small point or portion and contrast media filling up the tumour later, i.e. "progressive" enhancement (Fig. 1). Compared

respectively, after administration of gadopentetate dimeglumine, showed enhancement starting from one point of peripheral portion of the tumour (arrowhead). f Post-contrast T1-weighted imaging with fat saturation showed further enlargement of enhancement regions (arrowhead)

with the cavernous haemangiomas, all 16 schwannomas showed enhancement starting from a wide area and heterogeneous or homogeneous enhancement later (Fig. 2).

\section{Discussion}

Orbital cavernous haemangiomas, the most common primary orbital tumour in adults, demonstrate a predilection to affect middle-aged women (60-70\%), with a mean age of 43 to 48 years and range of 18 to 72 years based on several large series $[4,7,9]$. The most common presenting sign and symptom of the orbital cavernous haemangioma 
Table 1 Frequency distribution of non-enhanced MR imaging features

\begin{tabular}{|c|c|c|c|c|c|c|c|}
\hline \multirow[t]{3}{*}{ MR imaging feature } & \multicolumn{6}{|c|}{ Type of tumour } & \multirow[t]{3}{*}{$P$ value } \\
\hline & \multicolumn{2}{|c|}{ Overall } & \multicolumn{2}{|c|}{ Haemangioma } & \multicolumn{2}{|c|}{ Schwannoma } & \\
\hline & $N$ & $\%$ & $N$ & $\%$ & $N$ & $\%$ & \\
\hline No. of patients & 59 & & 43 & & 16 & & \\
\hline Anterior or posterior orbit & & & & & & & 0.004 \\
\hline Anterior & 47 & 79.6 & 39 & 90.8 & 8 & 50 & \\
\hline Posterior & 6 & 10.2 & 2 & 4.6 & 4 & 25 & \\
\hline Both & 6 & 10.2 & 2 & 4.6 & 4 & 25 & \\
\hline Extraconal or intraconal space & & & & & & & 0.049 \\
\hline Intraconal & 44 & 74.6 & 35 & 81.4 & 9 & 56.2 & \\
\hline Extraconal & 15 & 25.4 & 8 & 18.6 & 7 & 43.8 & \\
\hline Nasal or temporal orbit & & & & & & & 0.250 \\
\hline Nasal & 26 & 44.1 & 17 & 39.5 & 9 & 56.2 & \\
\hline Temporal & 33 & 55.9 & 26 & 60.5 & 7 & 43.8 & \\
\hline Superior or inferior orbit & & & & & & & 0.937 \\
\hline Superior & 29 & 49.2 & 21 & 48.8 & 8 & 50 & \\
\hline Inferior & 30 & 50.8 & 22 & 51.2 & 8 & 50 & \\
\hline Configuration & & & & & & & 0.015 \\
\hline Round & 6 & 10.2 & 4 & 9.3 & 2 & 12.6 & \\
\hline Ovoid & 50 & 84.7 & 39 & 90.7 & 11 & 68.7 & \\
\hline Irregular & 3 & 5.1 & 0 & 0 & 3 & 18.7 & \\
\hline Margin & & & & & & & 0.531 \\
\hline Smooth & 44 & 74.6 & 33 & 76.7 & 11 & 68.7 & \\
\hline Lobulated & 15 & 25.4 & 10 & 23.3 & 5 & 31.3 & \\
\hline T1 signal intensity ${ }^{a}$ & & & & & & & $<0.001$ \\
\hline Hypointense & 7 & 11.9 & 0 & 0 & 7 & 43.7 & \\
\hline Isointense & 52 & 88.1 & 43 & 100 & 9 & 56.3 & \\
\hline T1 homogeneity & & & & & & & $<0.001$ \\
\hline Homogeneous & 50 & 84.7 & 43 & 100 & 7 & 43.7 & \\
\hline Heterogeneous & 9 & 15.3 & 0 & 0 & 9 & 56.3 & \\
\hline T2 signal intensity ${ }^{\mathrm{a}}$ & & & & & & & 0.001 \\
\hline Isointense & 5 & 8.5 & 0 & 0 & 5 & 31.3 & \\
\hline Hyperintense & 54 & 91.5 & 43 & 100 & 11 & 68.7 & \\
\hline T2 homogeneity & & & & & & & $<0.001$ \\
\hline Homogeneous & 46 & 78 & 43 & 100 & 3 & 18.7 & \\
\hline Heterogeneous & 13 & 22 & 0 & 0 & 13 & 81.3 & \\
\hline
\end{tabular}

${ }^{a}$ Signal intensity was compared with that of extraocular muscle

is painless proptosis. In the past, orbital cavernous haemangiomas were invariably resected because they could not be conclusively discerned from other tumours clinically or radiologically [4]. However, orbital cavernous haemangiomas have recently been classified as type 3 low-flow arteriovenous malformations with a direct arterial in-flow and venous out-flow mechanism (arterial low-flow type) rather than as true neoplasms or proliferat- ing hamartomas [15], and, to our knowledge, there is no evidence of malignant transformation in the literature. Moreover, CT and MR imaging, especially MR imaging, have evolved to deliver high sensitivity and specificity in diagnosing orbital cavernous haemangiomas and distinguishing cavernous haemangiomas from other tumours, which have played a vital role in accurate diagnosis and correct treatment planning $[5,7,12,14,16]$. Therefore,

Table 2 Frequency distribution of contrast-enhanced MR imaging features

\begin{tabular}{|c|c|c|c|c|c|c|c|}
\hline \multirow[t]{3}{*}{ MR imaging feature } & \multicolumn{6}{|c|}{ Type of tumour } & \multirow[t]{3}{*}{$P$ value } \\
\hline & \multicolumn{2}{|c|}{ Overall } & \multicolumn{2}{|c|}{ Haemangioma } & \multicolumn{2}{|c|}{ Schwannoma } & \\
\hline & $N$ & $\%$ & $N$ & $\%$ & $N$ & $\%$ & \\
\hline No. of patients & 59 & & 43 & & 16 & & \\
\hline Spread pattern of contrast enhancement & & & & & & & $<0.001$ \\
\hline Starting from a small point or portion & 43 & 72.9 & 43 & 100 & 0 & 0 & \\
\hline Starting from a wide area & 16 & 27.1 & 0 & 0 & 16 & 100 & \\
\hline Enhancement pattern & & & & & & & $<0.001$ \\
\hline Homogeneous or heterogeneous & 16 & 27.1 & 0 & 0 & 16 & 100 & \\
\hline Progressive & 43 & 72.9 & 43 & 100 & 0 & 0 & \\
\hline $\mathrm{CI}$ & & & 1.8 & & 1.4 & & 0.003 \\
\hline TIC type & & & & & & & $<0.001$ \\
\hline Persistent & 12 & 20.3 & 3 & 7 & 9 & 56.2 & \\
\hline Plateau-shaped & 24 & 40.7 & 19 & 44.2 & 5 & 31.3 & \\
\hline Wash-out & 23 & 39 & 21 & 48.8 & 2 & 12.50 & \\
\hline
\end{tabular}



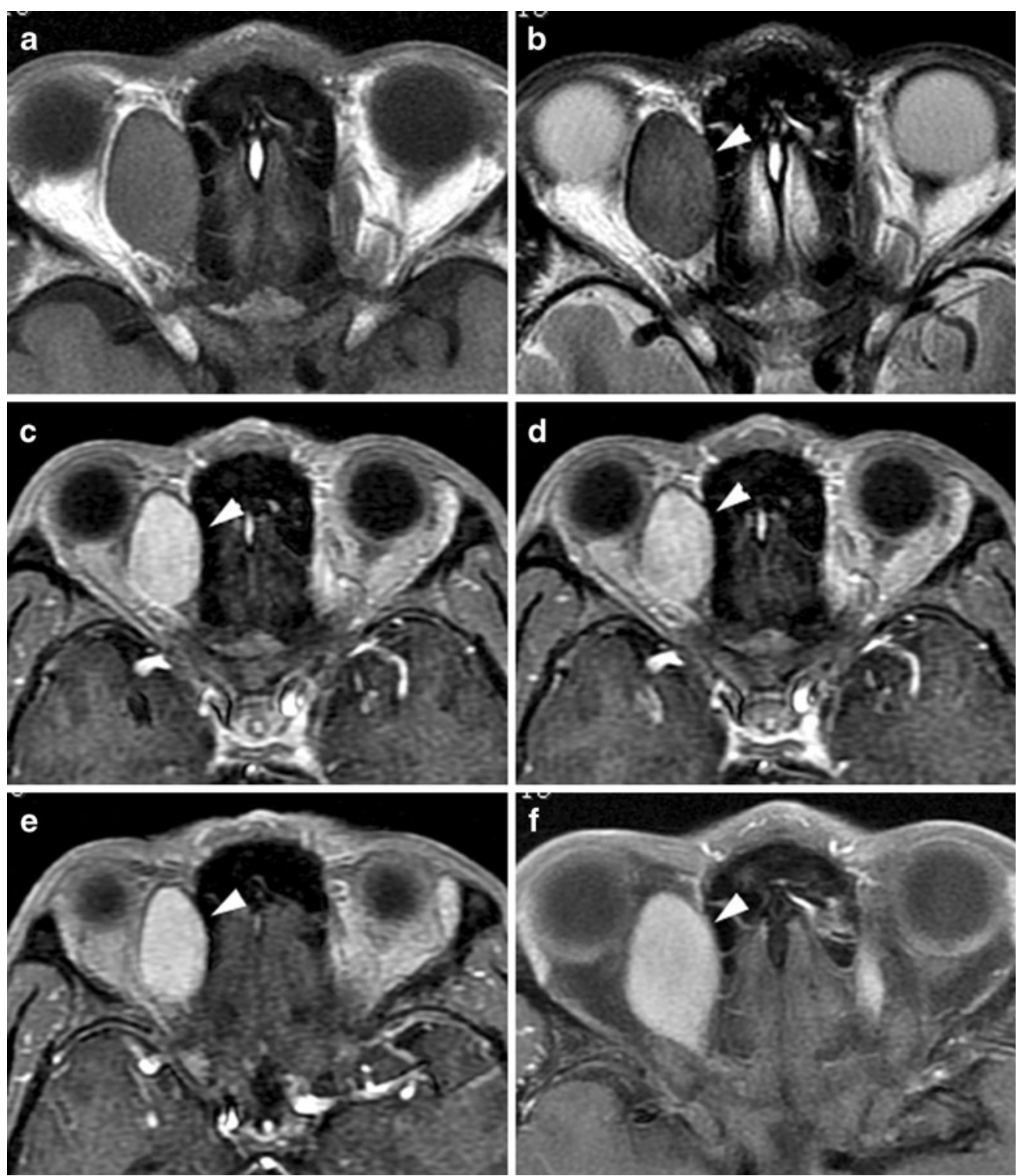

Fig. 2 A 42-year-old man with a schwannoma. a Axial T1-weighted imaging showed an oval extraconal isointense tumour relative to muscle. b Axial T2-weighted imaging revealed that the tumour was isointense relative to muscle (arrowhead). c-e Dynamic MR images obtained at 25, 75 and $225 \mathrm{~s}$, respectively, after administration of

recent literature supports observation as an alternative to surgical excision for asymptomatic orbital cavernous haemangiomas and surgery can be withheld until vision is threatened or cosmetic appearance resulting from proptosis is unacceptable [3,9].

Orbital schwannomas, constituting $1 \%$ of all orbital tumours, are benign peripheral nerve sheath tumours originating purely from Schwann cells and usually seen in young to middle-aged adults and rarely in children [3]. Clinical findings of orbital schwannomas are generally similar to those of cavernous haemangiomas, but they are somewhat more likely to exhibit progressive growth and symptoms. Thus, surgical excision is the treatment of choice for orbital schwannomas, different from orbital cavernous haemangiomas [3, 7-9].

gadopentetate dimeglumine showed enhancement starting from wide areas of tumour (arrowhead). f Axial post-contrast T1-weighted imaging with fat saturation showed homogeneous enhancement of the tumour (arrowhead)

Our results demonstrate that, in presence of a welldefined, ovoid, intraconal mass with markedly homogeneous hyperintensity signal on T2-weighted imaging, one small point or portion of enhancement initially noted after administration of contrast medium and "progressive" enhancement on later enhancement images, favours the diagnosis of cavernous haemangioma instead of schwannoma.

Most orbital cavernous haemangiomas are identified in the intraconal space, predominantly in the lateral aspect of the intraconal space [7,9]. In our series, only 8 of the 43 orbital cavernous haemangiomas were in the extraconal space. However, orbital schwannomas more commonly arise from sensory nerves in the orbit, specifically the supraorbital, supratrochlear and lacrimal nerves, and thus are 

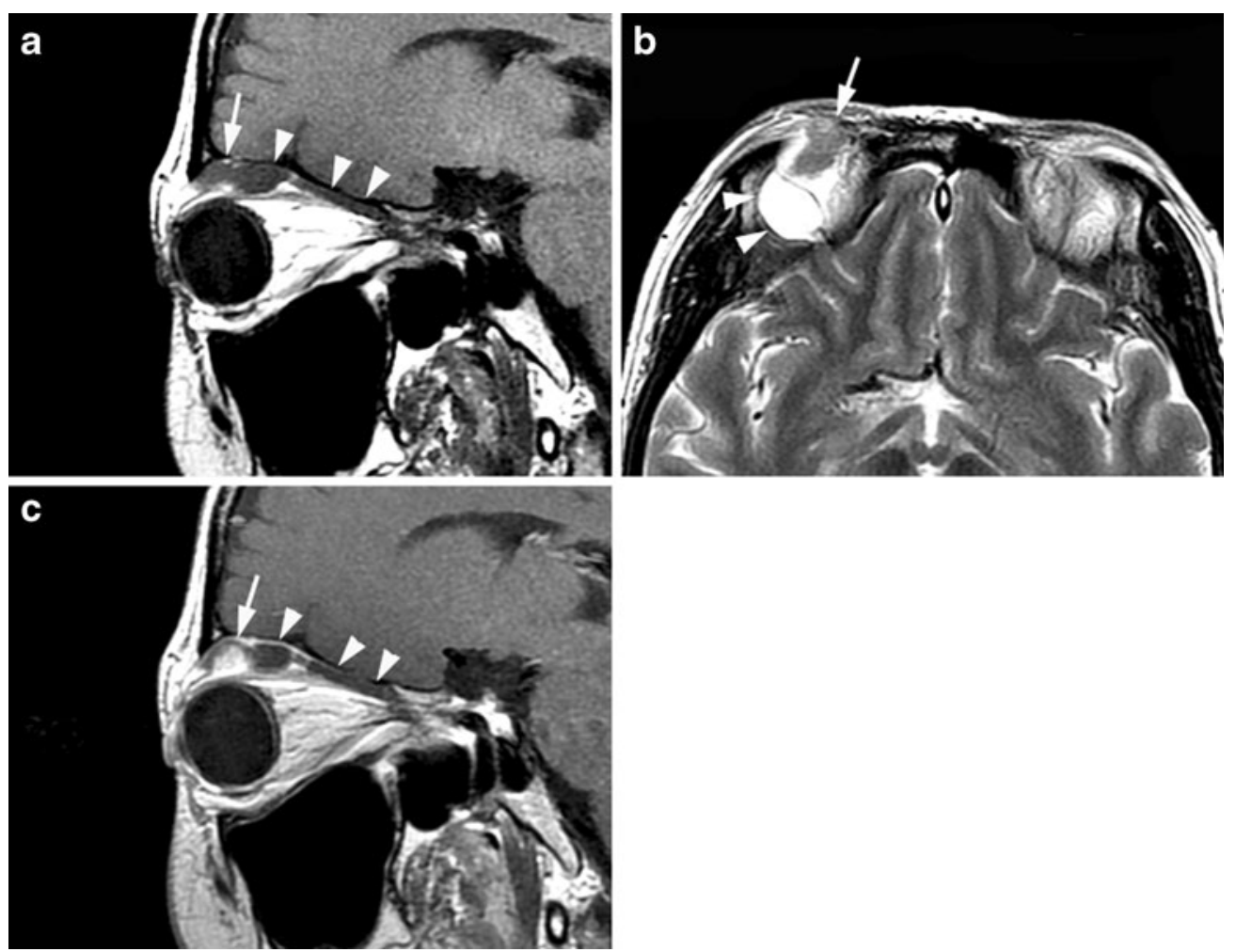

Fig. 3 A 47-year-old woman with schwannoma. a Obliquely sagittal T1-weighted imaging identified an irregular extraconal tumour with isointensity of the anterior portion of the tumour (arrow) and slight hypointensity of the posterior portion (arrowhead) relative to muscle. b Axial T2-weighted imaging showed isointensity of the anterior

usually outside the orbital muscle cone [16]. The nasociliary nerve is the only division of the ophthalmic nerve within the annular tendon [8]. Therefore, orbital schwannomas can be seen as an intraconal or extraconal mass. In our series, the frequency of the schwannoma being located in the extraconal or intraconal space is almost the same.

Orbital cavernous haemangiomas appear isointense to muscle on T1-weighted imaging and hyperintense to muscle on T2-weighted imaging [5, 7]. Although noncontrast MR imaging findings are non-specific, they may offer some advantage in the differentiation of orbital cavernous haemangiomas by demonstrating marked hyperintensity signal to muscle with internal septations on T2-weighted imaging, a hypointense circumferential rim corresponding to the fibrous pseudocapsule, and chemical shift artefact in the frequency-encoded direction secondary to high water content within the lesion surrounded by orbital fat $[5,7,17]$. Orbital schwannoma is isointense with respect to the extraocular muscle on T1weighted images and mildly hyperintense on T2-weighted images [6, 18]. Orbital schwannomas may undergo cavitary change, which appears as a cystic mass with straw-coloured fluid on gross pathological examination and looks homogeneously hypointense to muscle on T1weighted images and hyperintense on T2-weighted images $[8,19]$. Consequently, markedly homogeneous hyperintensity signal on T2-weighted imaging is one of the reliable findings distinguishing the two tumours.

portion of the tumour (arrow) and hyperintensity of the posterior portion (arrowhead) relative to muscle. c Obliquely sagittal postcontrast T1-weighted imaging demonstrated enhancement of the anterior portion of the tumour (arrow) and no enhancement of the posterior portion (arrowhead)

Variable degrees of contrast enhancement observed in orbital cavernous haemangiomas were reported, probably related to variable imaging times after contrast medium infusion. However, multiphase dynamic contrast CT and MRI demonstrated virtually pathognomonic imaging features of orbital cavernous haemangiomas [5, 7, 17]. On early imaging after the injection, one small point or portion of enhancement is initially noted because of its low-flow arterial supply, and the slow progressive accumulation of contrast medium continues within the dilated vascular spaces filling in centrally during the late venous phase. Subsequently, homogeneous contrast enhancement occurs and persists in the delayed equilibrium phases [7, 12-16]. This enhancement pattern is referred to as a "progressive" enhancement pattern in the literature [10, 11, 13]. Similar enhancement pattern is shared by cavernous haemangioma in the cavernous sinus and liver [20]. Several multiphase dynamic contrast MR imaging protocols have been reported for studying orbital cavernous haemangiomas [7, 11, 12, 14].

Compared with orbital cavernous haemangiomas, no "progressive" enhancement pattern was found in orbital schwannomas [12, 18]. Therefore, our results and the literature showed that the "progressive" enhancement pattern will be the most reliable finding in distinguishing orbital cavernous haemangiomas from schwannomas.

Some reports in the literature indicated that orbital cavernous haemangiomas showed an initial central point or 
portion enhancement after administration of gadopentetate dimeglumine [7, 12-14]. However, our results show that enhancement starts from one peripheral point or portion of the tumour in most orbital cavernous haemangiomas. The initial enhancement point represents the connecting point of feeding vessels to the lesion, which is useful for estimating the connecting point of the feeding vessels [14].

An unexpected result is that nearly half of orbital cavernous haemangiomas demonstrate a wash-out TIC type frequently seen in the malignant tumour rather than a plateau-shaped or persistent TIC type. This may be because contrast medium fills one portion of the tumour during an early phase after administration of contrast medium and contrast medium diffuses into adjacent spaces through the foramen of the fibrous interstitium between spaces at a later phase [12-14]. Thus it results in a decrease in concentration of contrast media in the portion of the tumour filled by contrast media during the early phase.

There is a significant difference in TIC type between orbital cavernous haemangiomas and schwannomas in contrast to a previous report that there is no significant difference in TIC type between the two tumours [12]. This is probably because we chose an ROI covering the greatest degree of early enhancement rather than the whole tumour. Recent literature on dynamic MR imaging in the breast, soft tissue and orbital masses indicates that an ROI covering the greatest degree of early enhancement of the mass reflects haemodynamic alterations of the tumour better than an ROI covering the whole tumour [10,21,22].

The multiphase dynamic contrast MR imaging findings of orbital cavernous haemangiomas have been described in the literature [10-14]. However, our results provided additional information on dynamic contrast-enhanced MR imaging findings of orbital cavernous haemangiomas. First, short imaging time and interval of every phase on dynamic contrast MR imaging may accurately show one point or small portion of enhancement of the mass on early phase images after the injection of contrast medium and allow characterization of specific spread pattern of the contrast enhancement in small orbital cavernous haemangiomas, which can differentiate small orbital cavernous haemangiomas from small schwannomas and other tumours. Second, TIC type of orbital cavernous haemangioma is significantly different from that of schwannoma.
Previous literature showed small haemangiomas could not be differentiated from haemangiopericytomas as their enhancement appears early and strongly and calcification could be noticed in cavernous haemangiomas, contributing to the differential diagnosis [5, 7]. However, calcification is occasionally seen in cavernous haemangiomas and plays a limited role in the differential diagnosis. Our results revealed that strong enhancement starting from a wide area and rapid wash-out on DCE MR imaging were seen in haemangiopericytomas that might be helpful in the differential diagnosis.

Capillary haemangiomas are a different sort of haemangiomatous tumour that are more common in children, often regress and have a different imaging appearance $[5,7]$.

\section{Conclusion}

Cavernous haemangiomas and schwannomas have different MR imaging features including the location, configuration and margins of the mass, signal intensity and homogeneity on T1- and T2-weighted imaging, the spread pattern of contrast enhancement, the enhancement pattern and TIC type, which could be helpful in the differentiation of the two tumours. The spread pattern of the contrast enhancement on dynamic contrast-enhanced MR imaging is the most reliable finding distinguishing cavernous haemangiomas and schwannomas. Markedly homogeneous hyperintensity signal on T2-weighted imaging is another reliable finding distinguishing the two tumours. MR imaging, especially dynamic contrast-enhanced MR imaging, plays a vital role in the differential diagnosis of cavernous haemangiomas and schwannomas.

Acknowledgements Junfang Xian and Zhengyu Zhang contributed equally to this article.

We would like to thank Drs. Jitong Shi and Bin Li of the Department of Ophthalmology, Beijing Tongren Hospital, for preparing clinical and pathological data.

Open Access This article is distributed under the terms of the Creative Commons Attribution Noncommercial License which permits any noncommercial use, distribution, and reproduction in any medium, provided the original author(s) and source are credited.

\section{References}

1. Shields JA, Bakewell B, Augsburger JJ, Flanagan JC (1984) Classification and incidence of space-occupying lesions of the orbit. A survey of 645 biopsies. Arch Ophthalmol 102:1606-1611

2. Moss HM (1962) Expanding lesions of the orbit. A clinical study of 230 consecutive cases. Am J Ophthalmol 54:761-770
3. Shields JA, Shields CL, Scartozzi R (2004) Survey of 1264 patients with orbital tumors and simulating lesions: the 2002 Montgomery Lecture, part 1. Ophthalmology 111:997-1008

4. Ruchman MC, Flanagan J (1983) Cavernous hemangiomas of the orbit. Ophthalmology 90:1328-1336

5. Bilaniuk LT (1999) Orbital vascular lesions. Role of imaging. Radiol Clin North Am 37:169-183
6. Gunduz K, Shields CL, Gunalp I, Erden E, Shields JA (2003) Orbital schwannoma: correlation of magnetic resonance imaging and pathology findings. Graefes Arch Clin Exp Ophthalmol 241:593-597

7. Ansari SA, Mafee MF (2005) Orbital cavernous hemangioma: role of imaging. Neuroimaging Clin North Am 15:137-158 
8. Kapur R, Mafee MF, Lamba R, Edward DP (2005) Orbital schwannoma and neurofibroma: role of imaging.

Neuroimaging Clin North Am 15:159-174

9. Scheuerle AF, Steiner HH, Kolling G, Kunze S, Aschoff A (2004) Treatment and long-term outcome of patients with orbital cavernomas. Am J Ophthalmol 138:237-244

10. Xian J, Zhang Z, Wang Z, Li J, Yang B, Man F et al (2010) Value of MR imaging in the differentiation of benign and malignant orbital tumors in adults. Euro Radiol. doi:10.1007/ S00330-009-1711-0

11. Thorn-Kany M, Delisle MB, Lacroix F, Lagarrigue J, Manelfe C (1999)

Cavernous hemangiomas of the orbit: MR imaging. J Neuroradiol 26:79-86

12. Tanaka A, Mihara F, Yoshiura T, Togao O, Kuwabara Y, Natori Y et al (2004) Differentiation of cavernous hemangioma from schwannoma of the orbit: a dynamic MRI study. AJR Am J Roentgenol 183:1799-1804
13. Wilms G, Raat H, Dom R, Carlo T, Philippe D, Godelieve D et al (1995) Orbital cavernous hemangioma: findings on sequential Gd-enhanced MRI. J Comput Assist Tomogr 19:548551

14. Ohtsuka K, Hashimoto M, Akiba H (1997) Serial dynamic magnetic resonance imaging of orbital cavernous hemangioma. Am J Ophthalmol 123:396-398

15. Rootman J (2003) Vascular malformations of the orbit: hemodynamic concepts. Orbit 22:103120

16. Mafee MF, Putterman A, Valvassori GE, Campos M, Capek V (1987) Orbital space-occupying lesions: role of computed tomography and magnetic resonance imaging. An analysis of 145 cases. Radiol Clin North Am 25:529559

17. Bilaniuk LT, Rapoport RJ (1994) Magnetic resonance imaging of the orbit. Top Magn Reson Imaging 6:167181

18. Xian J, Xu X, Wang Z, Yang B, Li B, Man F et al (2009) Magnetic resonance imaging findings of the uveal schwannoma. AJNR Am J Neuroradiol 30:769-773
19. Lam DSC, Ng JSK, To KF, Abdulah V, Liew CT, Tso MOM (1997) Cystic schwannoma of the orbit. Eye 11:798800

20. Wilms G (2009) Orbital cavernous hemangiomas. AJNR Am J Neuroradiol 30:E7

21. van Rijswijk CS, Geirnaerdt MJ, Hogendoorn PC, Taminiau AH, van Coevorden F, Zwinderman AH (2004) Soft-tissue tumors: value of static and dynamic gadopentetate dimeglumineenhanced MR imaging in prediction of malignancy. Radiology 233:493-502

22. Schnall MD, Blume J, Bluemke DA, DeBruhl N, Harms S, Heywang Kobrunner SH et al (2006) Diagnostic architectural and dynamic features at breast MR imaging: multicenter study. Radiology 238:42-53 